\section{Zometa ${ }^{\circledR}$ bei osteoblastischen Meta- stasen des Mammakarzinoms}

Ossäre Metastasen können als osteolytische, osteoblastische oder gemischte Formen auftreten. Sie führen häufig zu schwerwiegenden skelettalen Komplikationen wie pathologische Frakturen, Wirbelsäulenkompressionen oder Knochenschmerzen, die die Lebensqualität der betroffenen Patienten massiv beeinträchtigen. Bisphosphonate sind eine essenzielle Säule in der Therapie von Knochenmetastasen beim Mammakarzinom, so Professor Dr. Peyman Hadji, Marburg, auf dem Senologiekongress 2009. Da Bisphosphonate die osteoklastäre Aktivität hemmen, wird die metastasenbedingte Knochendestruktion reduziert. Die Bisphosphonat-Therapie wurde deshalb auch unter Progress von der Arbeitsgemeinschaft gynäkologische Onkologie (AGO) mit einer Grad A (++) Empfehlung bewertet [1].
Fast jede zweite Brustkrebs-Patientin mit Knochenmetastasen weist osteoblastische Anteile auf. Hier empfiehlt sich der Einsatz von Zoledronat $\left(\right.$ Zometa $^{\circledR}$ ), das Knochenkomplikationen nicht nur bei osteolytischen, sondern auch bei osteoblastischen Metastasen nachweislich verringert und verzögert [2]. In-vitro-Untersuchungen zeigten zudem, dass Zoledronat dosisabhängig die Osteoblastenzahl und -funktion reduziert [3]. Im Gegensatz dazu sind andere Bisphosphonate wie Pamidronat hier nicht wirksam [4]. Die monatlichen Infusionsintervalle der Substanz sind nach derzeitigem Wissensstand für eine effektive Osteoprotektion essenziell. Durch einfache und in den Alltag zu integrierende zahnmedizinische Vorsorgemaßnahmen vor einer Therapieeinleitung können Patientinnen mit Knochenmetastasen vor dem Auftreten einer Kieferosteonekrose während der Bisphosphonat-Therapie geschützt werden.

\section{Referenzen}

1 AGO-Empfehlungen 2009: http://www.ago-online.org/ index.php?lang $=$ de \&site $=$ mamma_guide_09_1 0\&topic $=$ mamma_guide

2 Saad F et al.: Long-term efficacy of zoledronic acid for the prevention of skeletal complications in patients with metastatic hormone-refractory prostate cancer. J Natl Cancer Inst 2004;96:879-82.

3 Orriss IR et al. Inhibition of osteoblast function by bisphosphonates. IBMS Poster-Präsentation \#143, 09.-14 März 2008, Davos.

4 Small EJ et al.: Combined analysis of two multicenter, randomized, placebocontrolled studies of pamidronate disodium for the palliation of bone pain in men with metastatic prostate cancer. J Clin Oncol 2003; 21:42774284.

Weitere Informationen bei

Hill \& Knowlton Communications GmbH

Holger Minning

Schwedlerstraße 6, 60314 Frankfurt

Tel. +49 69 97362-61, Fax -39

holger.minning@hillandknowlton.com

\section{In den Fokus stellen, was für die Patientin zählt}

Bei der Therapie des Mammakarzinoms steht neben der Verbesserung des Gesamtüberlebens in der adjuvanten Therapie und der Verlängerung der Überlebenszeit im metastasierten Stadium der Erhalt der Lebensqualität an vorderster Stelle. Unter dem Aspekt «Therapie des Mammakarzinoms - was zählt für die Patientin» beleuchtete der renommierte Gynäkologe Professor Wolfgang Janni von der Universitätsfrauenklinik Düsseldorf bei einem Meetthe-Expert-Symposium im Rahmen des Senologiekongresses 2009 aktuelle Daten zur adjuvanten endokrinen Therapie postmenopausaler Frauen.

Die Stellung der Aromatasehemmer als Stan- dardtherapie in dieser Indikation wurde aktuell von den Empfehlungen der AGO [1] und der St. Gallener Konsensuskonferenz [2] bestätigt. Sehr valide Daten aus einer SwitchEinzelstudie erbrachte die doppelblinde, internationale IES(IntergroupExemestaneStudy)Studie mit über 4700 Patientinnen, die den Wechsel auf Exemestan nach einer 2-3-jährigen adjuvanten Initialtherapie mit Tamoxifen im Vergleich zu einer 5-jährigen Tamoxifenbehandlung untersuchte [3]. Das Sterberisiko war in der Switch-Gruppe nach 52 Monaten um $23 \%$ ( $p=0,0069$, adjustiert nach prognostischen Faktoren) niedriger als in der TamoxifenGruppe [4]. Eine aktuelle Metaanalyse bestätigt die Überlebensvorteile für den Switch von Tamoxifen auf einen Aromatasehemmer: Für das sequenzielle Vorgehen zeigte diese Auswertung eine signifikante Reduktion der Gesamtmortalität im Zeitraum von 6 Jahren [5].

\section{Referenzen}

1 Therapieempfehlungen der Arbeitsgemeinschaft Gynäkologische Onkologie auf www.ago-online.org.

2 Konsensuskonferenz, St. Gallen Oncology Conference 2009.

3 Coombes RC et al.: Lancet 2007, 369:559-570

4 Fachinformation Aromasin ${ }^{\circledR}(05 / 2009)$.

5 Ingle JA et al.: San Antonio Breast Cancer Symposium 2008, Präsentation \#12.

Weitere Informationen bei Pfizer Deutschland GmbH

Stefanie Bockwinkel

Linkstraße 10, 10785 Berlin

Tel. +49 $30550055-52140$

stefanie.bockwinkel@pfizer.com

\title{
PharmaTicker+++ PharmaTicker+++ PharmaTicker+++ PharmaTicker+++
}

Mundipharma GmbH. Seit August 2009 gibt es $\operatorname{Targin}^{\circledR}$ in 4 Wirkstärken. Neben der 10 $\mathrm{mg} / 5 \mathrm{mg}$ und der $20 \mathrm{mg} / 10 \mathrm{mg}$ Retardtablette ist die Fixkombination aus retardiertem Oxycodon und retardiertem Naloxon dann auch als 5-mg/2,5-mg sowie 40-mg/20-mg-Dosierung verfügbar. Diese 4 flexiblen Dosierungsoptionen ermöglichen eine individuelle Schmerztherapie.

Dorothea Küsters Life Science Communications $\mathrm{GmbH}$ Petra Wollburg

Tel. +49 69 61998-23, Fax -10

wollburg@dkcommunications.de
GlaxoSmithKline GmbH. Onkologen profitieren zukünftig von einem noch breiteren Informationsangebot auf www.gsk-onkologie.de. GlaxoSmithKline (GSK) stellt innen sowohl Mitschnitte von Symposien zur Verfügung als auch Podcasts zu aktuellen onkologischen Themen. Mithilfe des Kongresskalenders sind Onkologen über Fachtreffen stets auf dem Laufenden.

GlaxoSmithKline GmbH \& Co. KG Arnd Prilipp

Tel. +49 89 36044-8117, Fax -8066 arnd.prilipp@gsk.com
Wyeth Pharma GmbH. Unter www.relistor.de startet das forschende Arzneimittelunternehmen Wyeth Pharma eine neue Website mit umfassenden Informationen rund um die Diagnose und Therapie der Opioid-induzierten Obstipation (OIC). Das Online-Angebot zur Diagnose und Therapie der OIC als Nebenwirkung der Schmerztherapie in der Palliativversorgung richtet sich an Ärzte und medizinisches Fachpersonal.

Wyeth Pharma Mirja Kaupmann

Tel. +49 251 204-1226, Fax -2033

kaupmaM@wyeth.com 
Phase-II-Studie beim fortgeschrittenen Brustkrebs:

\section{Positive Ergebnisse mit Nexavar ${ }^{\circledR}$ in Kombinationstherapie gegen Brust- krebs}

Das von Bayer HealthCare und Onyx Pharmaceuticals entwickelte Krebsmedikament Nexavar ${ }^{\circledR}$ (Wirkstoff: Sorafenib) zeigt in der Indikation Brustkrebs in Kombination mit oraler Chemotherapie vielversprechende Studienergebnisse. In einer ersten von einer unabhängigen Institution durchgeführten Phase-II-Studie bei fortgeschrittenem Brustkrebs wurde der primäre Endpunkt - eine Verbesserung der Überlebenszeit ohne Fortschreiten der Erkrankung - erreicht. Die Studie, an der 229 Patientinnen mit fortgeschrittenem oder metastasiertem HER-2-negativem (ohne Überexpression des HER-2-
Proteins) Brustkrebs teilnahmen, die vorher nicht mehr als eine Chemotherapie-Behandlung erhalten hatten, untersuchte Nexavar in Kombination mit dem Chemotherapeutikum Capecitabin. Der primäre Endpunkt der Studie war die Überlebenszeit ohne Fortschreiten der Erkrankung. Sekundäre Endpunkte beinhalteten das Gesamtüberleben, die Zeit bis zum Fortschreiten des Tumors sowie die Sicherheit. Die Patientinnen erhielten im 3wöchentlichen Zyklus randomisiert entweder oral 2-mal täglich $400 \mathrm{mg}$ Nexavar oder Placebo und zusätzlich das Chemotherapeutikum Capecitabin in der Dosierung $1000 \mathrm{mg} / \mathrm{m}^{2}$ Körperoberfläche 2-mal täglich über 14 Tage, gefolgt von einer 7-tägigen Einnahmepause von Capecitabin.

Die jetzt vorliegenden Ergebnisse der Studie zeigen, dass bei den mit Nexavar behandelten Patientinnen die mittlere Überlebenszeit bis zum Fortschreiten der Erkrankung gegenüber den Patientinnen, die nur die Chemotherapie erhielten, verlängert wurde. Diese Ergebnisse waren statistisch signifikant $(p-W e r t=0,0006)$. Sicherheit und Verträglichkeit im Kombinationsarm entsprachen den Erwartungen; neue oder unerwartete Toxizitäten traten nicht auf. Die detaillierten Ergebnisse der Studie sollen auf einem der nächsten wissenschaftlichen Kongresse vorgestellt werden.

Weitere Informationen bei

Bayer Schering Pharma AG

Frau Anna Koch

Müllerstraße 178, 13353 Berlin

Tel. + 4930 468-15942

anna.koch@bayerhealthcare.com
Optimiertes Management des HandFuß-Syndroms für eine erfolgreiche Capecitabin-Therapie bei metastasiertem Mammakarzinom

Das orale Fluoropyrimidin Capecitabin $\left(\right.$ Xeloda $\left.^{\circledR}\right)$ wird nach Anthrazyklin- und Taxan-Vorbehandlung zur Therapie des metastasierten Mammakarzinoms eingesetzt. Die hochwirksame Therapie bietet eine gut verträgliche Behandlungsalternative, die den Patientinnen eine höhere Lebensqualität ermöglicht. Sie gilt aufgrund ihrer Effektivität und guten Verträglichkeit als ein Mittel der Wahl, erkennbar an der ++-Empfehlung durch die AGO. Häufig tritt bei der Therapie mit Capecitabin lediglich ein Hand-Fuß-Syndrom (HFS) auf. Die geeigneten therapeutischen Maßnahmen zur Behandlung des HFS und die Ergebnisse wurden im Rahmen eines Satellitensymposiums anlässlich der 29. Jahrestagung der Deutschen Gesellschaft für Senologie vom 11.-13. Juni 2009 von Professor Andreas Schneeweiss, Heidelberg, präsentiert und diskutiert.

Die Therapie muss bei Auftreten eines HFS nicht komplett abgebrochen, sondern zunächst nur unterbrochen werden und kann nach $\mathrm{Ab}$ klingen der Symptome, gegebenenfalls mit einer reduzierten Dosis, wieder fortgesetzt werden. Eine auf Anforderungen der EMEA durchgeführte Metaanalyse vor allem bei metastasiertem Mamma- und metastasiertem kolorektalen Karzinom zeigte, dass das Auftreten eines HFS mit einer höheren Wahrscheinlichkeit für einen Therapieerfolg korrelierte. «Patienten mit einem HFS können auf
Basis dieser Erkenntnisse umso mehr motiviert werden, die Therapie nach einer Dosisanpassung fortzusetzen», erläuterte Schneeweiss.

Sowohl präventive, als auch lokale Maßnahmen helfen, die Symptome abzumildern oder das Auftreten des HFS komplett zu verhindern. Die umfassende Patientenaufklärung und eine konsequente Prophylaxe sind Hauptaspekte beim richtigen Management des HFS. Die Einbeziehung eines Dermatologen ist bei der Behandlung in jedem Fall ratsam.

Weitere Informationen bei

Roche Pharma AG

Dr. H.-U. Jelitto

Emil-Barell-Straße 1, 79639 Grenzach-Wyhlen Tel. +49 7624 14-2400, Fax -3366

www.roche.de

\section{PharmaTicker+++ PharmaTicker+++ PharmaTicker+++ PharmaTicker+++}

Sanofi-Aventis Deutschland GmbH. Die zulassungsrelevante Phase-III-Studie für BSI-201 in Kombination mit Chemotherapie bei Patientinnen mit metastasiertem triple-negativem Brustkrebs (mTNBC), definiert durch Tumoren ohne Expression von Östrogen- und Progesteronrezeptoren und ohne Überexpression von HER2, hat begonnen. Nähere Informationen über die klinische Phase-III-Studie sind unter www.clinicaltrials.gov oder www.biparsciences.com zu finden.

Sanofi-Aventis Deutschland GmbH Judith Kramer

Tel. +49 69 305-84412, Fax -18802

judith.kramer@sanofi-aventis.com medi GmbH. medi erweitert das Produktangebot neben der Kompressionsversorgung für die Extremitäten jetzt auf den Thorax in den Ausführungen mediven 550 Thorax Shirt, Bustier oder Body. Primäre und sekundäre Lymphödeme der Stadien I, II und III können mit der Thorax-Versorgung erfolgreich therapiert werden. Sie eignet sich auch bestens für Patientinnen, die neben stärksten Ödemen auch ein sehr weiches Bindegewebe aufweisen.

medi GmbH \& Co. KG

Tel. +49 921 912-381

www.medi.de
AstraZeneca GmbH. AstraZeneca und GWQ Service Plus AG kooperieren bei COMPACT: Im Rahmen des Projekts werden Real-Life-Daten in der adjuvanten Therapie mit dem Aromatasehemmer Anastrozol erfasst, die sowohl zur Verbesserung des Nebenwirkungsmanagements als auch zur gesundheitsökonomischen Bewertung einer modernen Therapie im Versorgungsalltag von Brustkrebspatientinnen herangezogen werden können.

AstraZeneca GmbH

Peter Schiffer

Tel. +49 4103 708-3663

peter.schiffer@astrazeneca.com 